\title{
Моделирование хромосферного излучения активных областей и сравнение с результатами наблюдений
}

\author{
К.А. Тлатова ${ }^{1}$, М.А. Лукичева ${ }^{2}$, В.В. Смирнова ${ }^{3}$ \\ 1 Кисловодская горная астрономическая станция ГАО РАН \\ k.tlatova@mail.ru \\ 2 Санкт-Петербургский государственный университет, математико-механический факультет \\ m.lukicheva@spbu.ru \\ 3 ГАО PAH \\ vosvid.smirnova@yandex.ru
}

Поступила в редакцию 16 ноября 2017 г.

Аннотация. В работе представлены результаты моделирования хромосферного излучения активных областей с использованием современной интерактивной системы трехмерного моделирования GX Simulator. Синтезированные карты яркостной температуры сравниваются с результатами наблюдений радиоинтерферометров Atacama Large Millimeter/Submillimeter Array (ALMA) и Nobeyama Radioheliograph (NoRH).

MODELING OF THE CHROMOSPHERIC RADIATION OF ACTIVE REGIONS AND COMPARISON WITH RESULTS OF OBSERVATIONS, by K.A. Tlatova, M.A. Loukitcheva, V.V. Smirnova. The article presents the results of simulating the chromospheric radiation of active regions using the modern interactive 3D modeling framework GX Simulator. The synthesized maps of brightness temperature are compared with the results of observations of the Atacama Large Millimeter/Submillimeter Array (ALMA) and Nobeyama Radioheliograph (NoRH).

Ключевые слова: Солнце, хромосфера, радиоизлучение, активные области

\section{1 Введение}

Наблюдения солнечных активных областей (AO) на миллиметровых (мм) волнах радиодиапазона имеют полувековую историю, начавшуюся еще в семидесятых годах двадцатого века (Нагнибеда и Лукичева, 2016). Первые наблюдения АО проводились на одиночных параболических антеннах РТ-7.5 МГТУ им. Баумана, РТ-14 обсерватории Метсахови и РТ-22 КрАО. К сожалению, большая ширина диаграммы направленности одиночных антенн, составляющая несколько угловых минут, и, как следствие, низкое угловое разрешение инструментов не позволяют использовать данные инструменты для детального исследования солнечной хромосферы, из которой исходит регистрируемое миллиметровое излучение. На картах интенсивности, получаемых на одиночных антеннах, активным областям соответствуют области повышенной над уровнем спокойного Солнца радиояркости, совпадающие по размеру с окружающим флоккулом. 
Возможности исследования $\mathrm{AO}$ по наблюдениям на мм волнах значительно расширились с появлением интерферометрических систем наблюдений. Для интерферометров главным фактором, определяющим пространственное разрешение, является максимальное расстояние, на которое разнесены антенны. При этом качество получаемого изображения зависит от количества антенн, составляющих интерферометрическую систему, и способа заполнения апертуры интерферометра. Первые интерферометрические изображения Солнца на мм волнах были получены в 2003-2004 гг. с использованием интерферометра Berkeley-Illinois-Maryland Array (BIMA) на волне 3.5 мм и с пространственным разрешением 12" (Уайт и др., 2006; Лукичева и др., 2014). На полученных хромосферных изображениях пятну соответствовала область самых низких яркостных температур на карте $\mathrm{AO}$, с яркостью ниже, чем в областях спокойного Солнца. Другим примером успешного наблюдения $\mathrm{AO}$ на коротких волнах радиодиапазона являются карты интенсивности, полученные на интерферометре NoRH на волне 8 мм с разрешением $5^{\prime \prime}$ (Иваи и др., 2016), на которых радиояркость в области пятна также не превышает уровня спокойного Солнца.

В 2014-2015 гг. были проведены первые наблюдения солнечных пятен на интерферометре ALMA, являющимся самым крупным наземным телескопом (Хиллс и др., 2010). Система ALMA обладает уникальными для данного диапазона характеристиками и позволяет наблюдать радиоисточники с разрешением до долей угловых секунд. Первые изображения AO с помощью системы ALMA были получены в ходе солнечных тестовых кампаний 2014-2015 гг. на волнах 1 и 3 мм (Шимоджо и др., 2017). Для тестовых наблюдений $\mathrm{AO}$ была использована конфигурация из 36 антенн, включая антенны основной решетки ALMA, антенны компактной решетки Atacama Compact Array (ACA) и антенны для измерения полного потока Total Power Array (ТP). Пространственное разрешение полученных в такой конфигурации изображений составило (2-4)" для 3 мм и (1-2)" для 1 мм, что позволяет исследовать хромосферу АО с детальностью, недоступной ранее.

\section{2 Наблюдательные данные и модели}

В качестве наблюдательных данных для сравнения с результатами моделирования мы использовали изображения активной области AR 12470, полученные с помощью интерферометра ALMA на волне 3 мм (частота 100 ГГц) 16.12.2015, а также полученные 18.12.2015 на волне 1 мм (230 ГГц). Изображения ALMA представляют собой мозаику из 149 элементов, при этом поле зрения на волне 3 мм составляет $300 \times 300^{\prime \prime}$, а на 1 мм - $140 \times 140^{\prime \prime}$. Пространственное разрешение анализируемых карт составило $(4.9 \times 2.2)^{\prime \prime}$ и $(2.4 \times 0.9)^{\prime \prime}$ соответственно. На волне 3 мм в данной АО было обнаружено повышение радиояркости в центральной части тени пятна, которое составило $\sim 800 \mathrm{~K}$ (Иваи и др., 2017). В изображении на 1 мм наблюдается депрессия излучения в центральной части тени пятна на $\sim 700 \mathrm{~K}$ относительно уровня спокойного Солнца (Лукичева и др., 2017).

В данной работе мы использовали пакет GX Simulator (Нита и др., 2015, 2017) как интерактивную среду для моделирования радиоизлучения хромосферы. Изначально GX Simulator был разработан для моделирования вспышечных процессов в короне, при этом моделирование хромосферы было представлено в упрощенном виде. В обновленной версии GX Simulator был модернизирован с целью более реалистичного описания хромосферных слоев, в результате чего стало доступно моделирование как хромосферы, так и короны в полном объеме (Нита и др., 2017). В ходе модернизации среды моделирования был разработан новый подход к заполнению хромосферного объема трехмерной модели. Для этой цели первоначально производится фрагментация диска на яркостные компоненты, затем создаются маски выделенных компонент, в конце происходит заполнение хромосферного объема одномерными (1D) моделями на основании полученных масок. При этом возможно использование широкого набора 1D-моделей. В данной работе были апробированы 2 набора моделей, именуемых в дальнейшем как Метод 1 и Метод 2 и описанных ниже.

В качестве исходных данных для моделирования мы использовали фотогелиограммы (изображения в белом свете) и магнитограммы, получаемые инструментом Helioseismic and Magnetic Imager (HMI, Шеррер и др., 2012) со спутника Solar Dynamics Observatory (SDO). Пример фотосферных данных, используемых при моделировании, представлен на рис. 1. На основе значений магнитограммы продольной компоненты магнитного поля происходит разделение областей спокойного Солнца и 

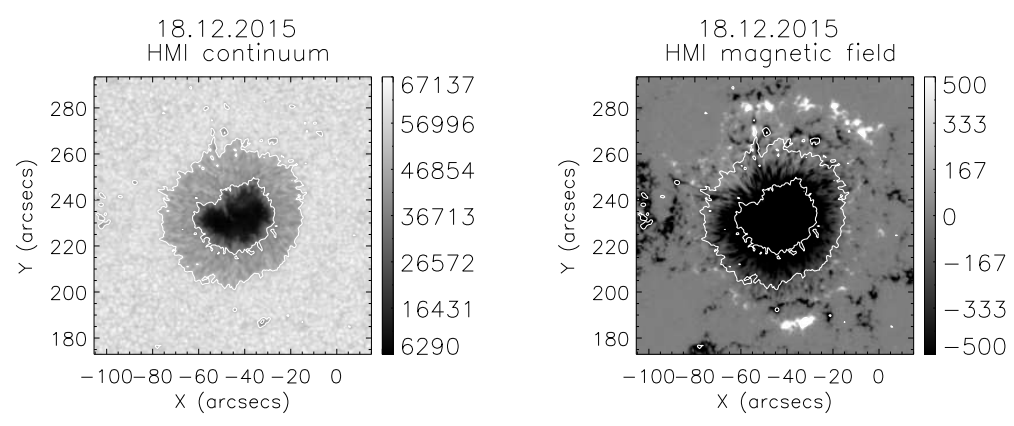

Рис. 1. Фотогелиограмма (слева) и магнитограмма (справа) для AR 12470, полученные по данным HMI/SDO 18.12.2015 в 19:30 UT. Белые контуры соответствуют границам тени и полутени пятна. Для усиления контраста значения магнитограммы отображены в диапазоне $[-500,500]$ Гс. Максимальное значение напряженности магнитного поля пятна на уровне фотосферы составило -2800 Гс

активных областей. Дальнейшая фрагментация производится на основе значений отсечки для разных яркостных компонент на гистограмме интенсивности в белом свете, после чего создаются маски и происходит заполнение хромосферного объема одним из выбранных методов. Для Метода 1 был использован набор из семи классических 1D полуэмпирических моделей яркостных компонент спокойного и активного Солнца, разработанный Фонтенла и др. (2006, 2009, 2011). Данные модели описывают распределение температуры и плотности с высотой в хромосфере для следующих яркостных элементов: тень и полутень пятна, узлы и границы магнитной сетки, усиленная магнитная сетка, а также факельная и флоккульная компоненты. Модели разных яркостных компонент отличаются градиентами температуры и плотности в хромосфере, а также высотой, на которой расположена переходная область от хромосферы к короне. В Методе 2 набор 1D-моделей Фонтенла и др. был расширен и дополнен 3D-кубом РМГД-моделей, полученных с помощью кода Bifrost (Карлссон и др., 2016); данный куб используется в качестве репозитория для дополнительных моделей разных яркостных компонент.

\section{3 Результаты моделирования}

Для даты 18.12.2015 моделирование исходящего хромосферного излучения было проведено на трех частотах: 230 ГГц, 34 ГГц и 17 ГГц двумя описанными выше методами. При расчете исходящего излучения учитывались тепловой тормозной и гирорезонансный механизмы генерации излучения. Далее модельные распределения интенсивности были свернуты с диаграммой направленности (ДН) инструментов. Для оценки проведенного моделирования полученные модельные распределения сравнивались с результатами наблюдений на ALMA (на 230 ГГц) и с наблюдениями на NoRH (на 34 ГГц и 17 ГГц). Результаты расчетов и наблюдений представлены на рис. 2. Модельные распределения на 230 Ггц показывают депрессию в области тени пятна, что согласуется с наблюдательными данными на данной волне. Однако на более низких частотах, 34 ГГц и 17 ГГц, не наблюдается полного соответствия между наблюдениями и моделированием, при этом результаты моделирования различными методами значительно различаются между собой. При моделировании Методом 1 на обеих частотах над пятном имеется яркий источник, в то время как Метод 2 показывает депрессию излучения над пятном. Однако наблюдения свидетельствуют о депрессии на 34 ГГц и ярком источнике на 17 ГГц, но сдвинутом относительно центра пятна. Данный источник на 17 ГГц можно также интерпретировать как уярчение полутени, что согласуется с моделью по Методу 2. В целом наглядно видно, что Метод 1 недостаточно реалистично описывает наблюдения активной области. 

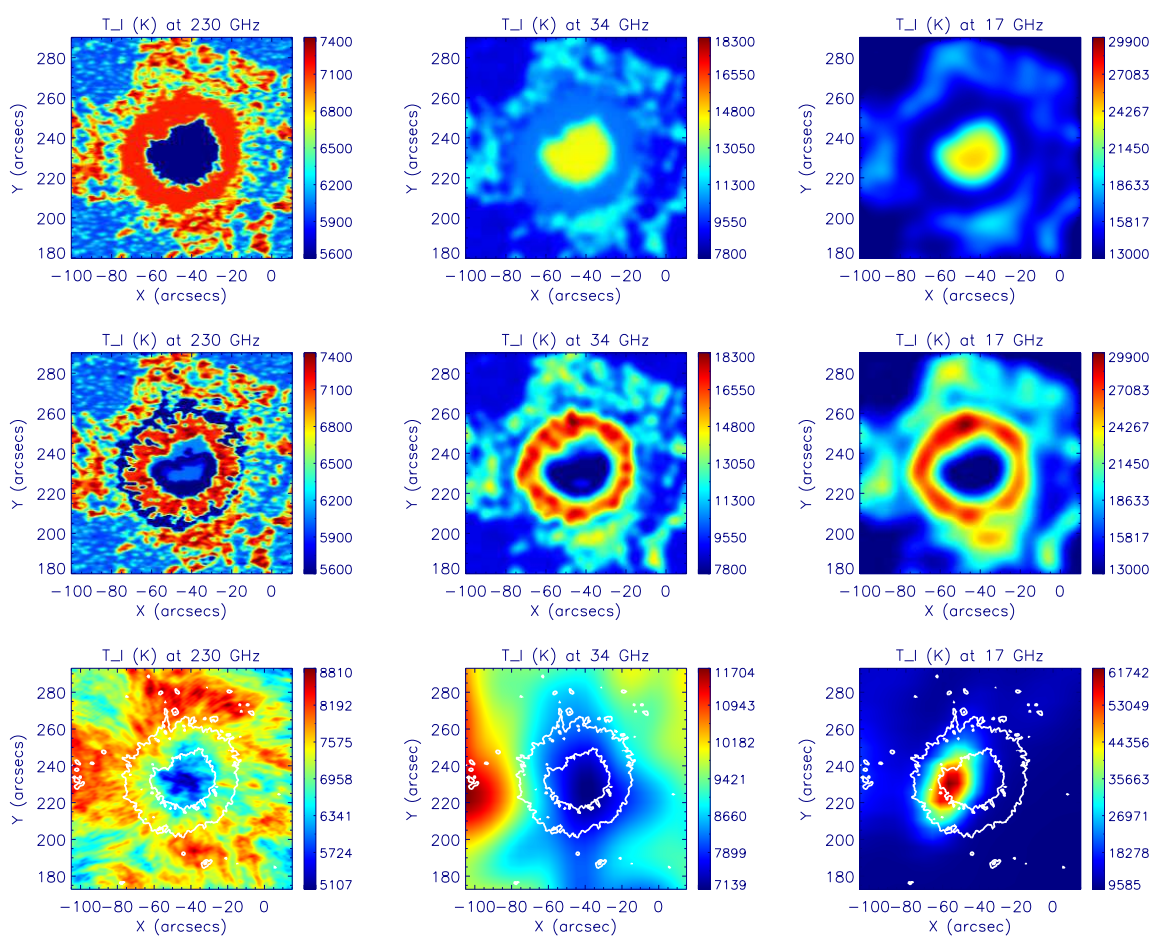

Рис. 2. Результаты моделирования по Методу 1 (сверху) и Методу 2 (в середине), представленные в виде карт яркостной температуры, свернутых с ДН-инструментов, на частотах 230 ГГц, 34 ГГц, 17 ГГц. Нижняя панель: наблюдательные карты AR 12470 на частотах 230 ГГц (по данным ALMA), 34 ГГц и 17 ГГц (по данным NoRH). Белые контуры соответствуют границам тени и полутени пятна
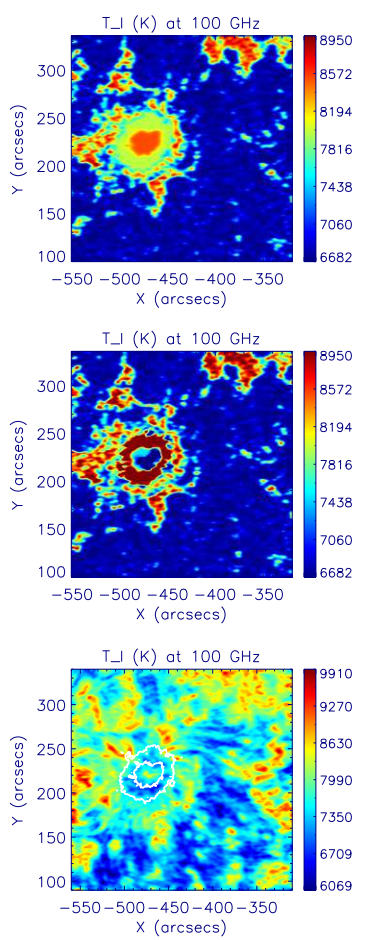
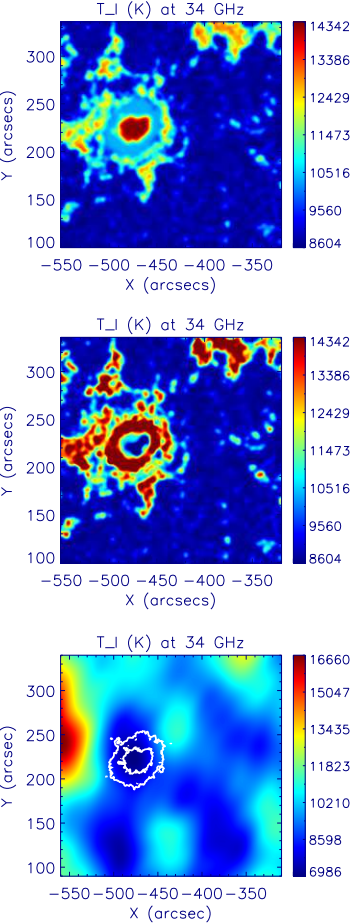
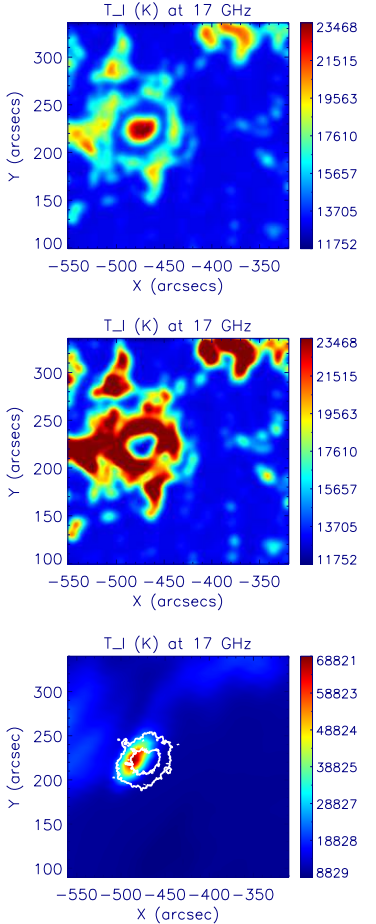

Рис. 3. То же, что на рис. 2 для 16.12 .2015 и частот 100 ГГц, 34 ГГц и 17 ГГц 
Для даты 16.12.2015 моделирование было проведено на частотах 100 ГГц, 34 ГГц и 17 ГГц аналогично предыдущему случаю двумя описанными выше методами, а затем полученные карты были свернуты с диаграммой направленности. Полученные модельные распределения сравнивались на 100 ГГц с наблюдениями на ALMA и на 34 ГГц и 17 ГГц с наблюдениями на NoRH (рис. 3). На карте, полученной Методом 1 на 100 ГГц, имеется уярчение в области тени пятна, что соответствует наблюдениям. На 34 ГГц Метод 2 показал депрессию тени пятна, что также согласуется с результатами наблюдений. На 17 ГГц, как и в случае для 18.12.2015, в наблюдениях источник оказался сдвинут относительно центра пятна.

Таким образом, ни первым, ни вторым методом мы не получили полного соответствия между наблюдениями и результатами моделирования активной области. При использовании Метода 1 наблюдается уярчение в области тени солнечного пятна, а при Методе 2 , напротив, имеет место холодная (темная) область. Рассмотренные нами модели не удовлетворяют одновременно многоволновым данным, согласие между наблюдениями и моделированием удается достигнуть только частично.

\section{4 Выводы}

Миллиметровые наблюдения активных областей с высоким разрешением несут важную информацию о тепловой структуре хромосферы. В связи с этим одной из важнейших задач является построение реалистичных моделей хромосферы активных областей. Мы использовали современный инструмент GX Simulator для синтезирования результатов планируемых наблюдений и тестирования различных моделей. В рамках рассмотренных моделей и методов заполнения хромосферного объема нам не удалось построить тепловую структуру хромосферы, которая позволяет одновременно описать совокупность наблюдений АО на трех волнах мм диапазона. Очевидно, что набора моделей из 7 компонент, используемого в Методе 1, недостаточно для реалистичного описания хромосферы. Кроме этого, для моделирования наблюдений на 17 ГГц (NoRH), для которых характерно наличие яркого источника, не совпадающего по положению с центром пятна, необходимо использование дополнительных критериев при заполнении хромосферного объема модели, а именно включение не только фотосферных, но и хромосферных масок яркостных компонент.

Благодарности. Работа выполнена при поддержке гранта РФФИ 16-02-00749.

\section{Литература}

Иваи и др. (Iwai K., Koshiishi Н., Shibasaki K.) // Astrophys. J. 2016. V. 816. P. 91.

Иваи и др. (Iwai K., Loukitcheva M., Shimojo M., Solanki S.K., White S.M.) // Astrophys. J. Lett. 2017. V. 841. P. L20.

Карлссон и др. (Carlsson M., Hansteen V., Gudiksen B., Leenaarts J., De Pontieu B.) // Astron. Astrophys. 2016. V. 585. P. A4.

Лукичева и др. (Loukitcheva M., Solanki S.K., White S.M.) // Astron. Astrophys. 2014. V. 561. P. A133.

Лукичева и др. (Loukitcheva M., Iwai K., Solanki S.K., White S., Shimojo M.) // Astrophys. J. 2017. V. 850. P. 35

Нагнибеда В.Г., Лукичева М.А. // Изв. Крымск. Астрофиз. Обсерв. 2016. Т. 112. № 2. С. 167.

Нита и др. (Nita G., Fleishman G., Kuznetsov A., Kontar E., Gary D.) // Astrophys. J. 2015. V. 799. Р. 236.

Нита и др. (Nita G., Viall N., Klimchuck J., Loukitcheva M., Gary D., Kuznetsov A., Fleishman G.) // Astrophys. J. 2018. V. 853. Issue 1. id. 66.

Шеррер др. (Scherrer P.H., Schou J., Bush R.I., et al.) // Solar Phys. 2012. V. 275. P. 207.

Уайт и др. (White S.M., Loukitcheva M., Solanki S.K.) // Astron. Astrophys. 2006. V. 456. P. 697.

Фонтенла и др. (Fontenla J.M., Avrett E., Thuillier G., Harder J.) // Astrophys. J. 2006. V. 639. P. 441. 
Фонтенла и др. (Fontenla J.M., Curdt W., Haberreiter M., Harder J., Tian H.) // Astrophys. J. 2009. V. 707. P. 482.

Фонтенла и др. (Fontenla J.M., Harder J., Livingston W., Snow M., Woods T.) // J. Geophys. Res. Atmos. 2011. V. 116. P. D20108.

Хиллс и др. (Hills R.E., Kurz R.J., Peck A.B.) // Proc. SPIE. 2010. V. 7733. id. 773317.

Шимоджо и др. (Shimojo M., Bastian T.S., Hales A.S., et al.) // Solar. Phys. 2017. V. 292. P. 87. 